\title{
BMJ Open Perspectives and experiences of collecting antenatal colostrum in women who have had diabetes during pregnancy: a North Queensland semistructured interview study
}

Jordan Rita Rose Casey, ${ }^{1}$ Erin Louise Mogg, ${ }^{1}$ Jennifer Banks, ${ }^{1}$ Kathleen Braniff, ${ }^{2}$ Clare $\mathrm{Heal}^{1}$

To cite: Casey JRR, Mogg EL, Banks J, et al. Perspectives and experiences of collecting antenatal colostrum in women who have had diabetes during pregnancy: a North Queensland semistructured interview study. BMJ Open 2019;9:e021513. doi:10.1136/ bmjopen-2018-021513

\section{- Prepublication history for} this paper is available online. To view these files, please visit the journal online (http://dx.doi. org/10.1136/bmjopen-2018021513).

Received 5 January 2018 Revised 12 July 2018 Accepted 23 October 2018

Q Check for updates

(c) Author(s) (or their employer(s)) 2019. Re-use permitted under CC BY-NC. No commercial re-use. See rights and permissions. Published by BMJ.

1James Cook University College of Medicine and Dentistry, Mackay, Queensland, Australia ${ }^{2}$ Women's Health Unit, Mackay Base Hospital, Mackay, Queensland, Australia

Correspondence to Dr Jordan Rita Rose Casey; jordan.casey2@my.jcu.edu.au

\section{ABSTRACT}

Objectives To explore and describe the experiences and perspectives of collecting and storing colostrum in the antenatal period in women who have had diabetes in pregnancy.

Design Face-to-face, semistructured interviews analysed with purposive sampling and thematic analysis.

Setting A regional hospital in North Queensland with a high prevalence of diabetes in pregnancy.

Participants Six women with a previous pregnancy complicated by diabetes who were advised to collect and store colostrum in pregnancy.

Results Six themes were identified: wariness of medicalisation (adjusting to an 'abnormal' pregnancy, seeking continuity of care, determination to reduce formula, fear of invasive intervention); underlying altruism (providing the best for baby, preparing for complications, eager for milk donation); internal pressure to succeed (coping with confronting information, disheartened by failures, constant fear of insufficient supply, overwhelming guilt, concern for future breastfeeding success); selfmanagement and ownership (adapting to awkwardness, developing strategies for success, actively seeking education, gaining confidence to request help, accepting personal limitations); frustrated by waste (encroaching on time, squandering a precious resource, ambiguous about necessity) and building fortitude for motherhood (physically preparing for breast feeding, symbolic of the imminent infant, establishing early relationships with supports, approaching challenges with realistic optimism). Conclusion Women with diabetes in pregnancy experience guilt and stress about the added risk of hypoglycaemia to their babies and strive to provide the best for their babies by collecting and storing colostrum, even if this leads to distress to themselves. It is crucial that these women be provided accurate, realistic advice about the benefits and disadvantages of collecting colostrum in the antenatal period.

\section{BACKGROUND}

Diabetes mellitus is a common problem that affects more than $13 \%$ of pregnant women
Strengths and limitations of this study

- In-depth, face-to-face interviewing allowed for a detailed understanding of women's experiences of collecting and storing colostrum.

- Participants were recruited until theoretical saturation was achieved.

- Researcher triangulation was used to ensure that the themes reflected the range and depth of data collected.

- A limitation of this study was that only Caucasian women were represented in the sample.

- The study was only conducted in one centre, so transferability to other settings is uncertain.

in Australia. ${ }^{1}$ Diabetes in pregnancy includes both gestational diabetes mellitus (GDM) and pre-existing diabetes (type I diabetes mellitus or type II diabetes mellitus). Diabetes in pregnancy is associated with multiple risks to the newborn including macrosomia, shoulder dystocia, polycythaemia, hyperbilirubinaemia and neonatal hypoglycaemia. ${ }^{2}$ Hypoglycaemia is a significant problem as it is associated with cardiorespiratory disturbances, damage to the central nervous system and developmental delay. ${ }^{3}{ }^{4}$ Approximately 6\% of Australian neonates experience hypoglycaemia; however, this rate is greatly increased in infants born to mothers with diabetes with $25 \%-50 \%$ of newborns affected. ${ }^{5}$ The mainstay of management of infants at risk of hypoglycaemia is early breast feeding and top-up feeding with colostrum or formula. ${ }^{6}$

Some women with diabetes in pregnancy are referred to a lactation consultant and encouraged to begin expressing and storing colostrum in the late antenatal period. ${ }^{7}$ This practice has been thought to promote earlier onset of lactogenesis and facilitate 
early breast feeding, as well as provide a supply of colostrum that may be used to supplement breast feeding and reduce reliance on formula feeding to treat hypoglycaemia. ${ }^{78}$ Because of these perceived benefits to the neonate, women with diabetes in pregnancy are encouraged to collect and store colostrum by midwives, diabetes educators and obstetricians in many settings. ${ }^{9}$ However, the experiences of women with diabetes who are encouraged to engage in this practice have not been well explored.

The Diabetes and Antenatal Milk Expressing (DAME) study, a multicentre randomised controlled trial in Melbourne, found that expressing from 36 weeks of gestation was safe in low-risk women with diabetes and may have some benefits to the neonate including increased rates of exclusive breast feeding in the first 24 hours of life. ${ }^{10}$ In women without diabetes in pregnancy, antenatal expressing has also been shown to be associated with reduced time to initiation of full establishment of lactation, which suggests expressing in the antenatal period may improve postnatal lactational performance. ${ }^{8}$ However, a qualitative study of antenatal expressing in women without diabetes in pregnancy found that though collecting colostrum antenatally may increase confidence and breast familiarity, it can also evoke embarrassment and can be difficult and painful to engage in. ${ }^{11}$

Women with diabetes in our centre routinely attend an education session with the diabetes educator midwife, where they are advised to collect antenatal colostrum and are referred to a lactation consultant. Women are provided with syringes and labels and are encouraged to begin expressing colostrum daily from 34 to 36 weeks of gestation and continue until birth.

This study aims to explore the perspectives and experiences of women who have had diabetes in pregnancy and were encouraged to collect and store colostrum in the antenatal period, which may elucidate motivating factors, challenges, benefits, and stress factors in this population.

\section{METHODS}

The Consolidated Criteria for Reporting Qualitative Health Research (COREQ) was followed. ${ }^{12}$

\section{Patient selection and setting}

Women were eligible to participate in this study if they were English speaking, had experienced diabetes in pregnancy, had attended an education session with the diabetes educator midwife about collecting antenatal colostrum, had had a live, singleton birth and were at least 6 weeks postpartum. Purposive sampling was used to capture a range of clinical characteristics including type of diabetes (GDM or diabetes unrelated to pregnancy) and neonatal complications. Selected women who had been registered on the 2014 and 2015 diabetes antenatal register at the study hospital were identified by the diabetes educator midwife, contacted by mail and invited to participate. Participants who returned the signed consent form were contacted by the primary author (JRRC) to arrange an interview.

\section{Data collection}

A semistructured face-to-face interview was conducted with each participant in the women's choice of their home, meeting room at the hospital or local café between May 2017 and July 2017. The average length of interview was $28 \mathrm{~min}$. All interviews were conducted by one author, who is a female medical student, experienced in qualitative research (JRRC). No participants were known to the interviewer. Participants were alone or accompanied by their infant. The interview guide included questions about experiencing and coping with diabetes in pregnancy, expressing and storing colostrum, postpartum adjustment and breast feeding. This guide was developed after a review of the literature ${ }^{7-11}$ and discussion among the investigators, which consisted of an obstetric clinician and research officers experienced in qualitative research. Interviews were digitally recorded with participants' consent and transcribed. Field notes were also written during the interviews. Interviews were conducted until theoretical saturation was achieved.

\section{Patient and public involvement}

No patients were directly involved in the development of the research question, selection of the outcome measures, design of the study or interpretation of the results.

\section{Data analysis}

Transcripts were entered in software for qualitative data management (HyperRESEARCH; V.3.5.2 ResearchWare) for analysis. Drawing on the principles of grounded theory and thematic analysis, one author (JRRC) performed line-by-line coding of each transcript to inductively identify themes in the data (ie, themes were not established a priori).$^{13}$ New codes were created to capture new concepts. The preliminary themes and codes were reviewed by three other authors (ELM, JB, CH) and iterated to ensure that codes and themes reflected the data collected (investigator triangulation). Conceptual patterns and links between themes were identified to develop a thematic schema.

\section{RESULTS}

\section{Participant characteristics}

Thirty participants were contacted by mail. Six participants consented to be interviewed. Participant characteristics are provided in table 1 . The age of participants ranged from 27 to 45 years. Of the six participants, five had had GDM and one had type I diabetes mellitus. All six women were diagnosed with diabetes during pregnancy. Though all attempted to collect antenatal colostrum, five of the participants were able to obtain colostrum. Four of the participants had babies who experienced neonatal hypoglycaemia. 


\begin{tabular}{|c|c|}
\hline Characteristics & Total $\mathbf{n}$ \\
\hline \multicolumn{2}{|l|}{ Age group (years) } \\
\hline $25-30$ & 2 \\
\hline $31-35$ & 3 \\
\hline$>35$ & 1 \\
\hline \multicolumn{2}{|l|}{ Ethnicity } \\
\hline Caucasian & 6 \\
\hline \multicolumn{2}{|l|}{ Marital status } \\
\hline Married & 5 \\
\hline De facto & 1 \\
\hline \multicolumn{2}{|l|}{ Education level } \\
\hline Grade 12 or equivalent & 1 \\
\hline Diploma & 2 \\
\hline Bachelor's degree & 2 \\
\hline Postgraduate degree & 1 \\
\hline \multicolumn{2}{|l|}{ Diabetes type } \\
\hline GDM & 5 \\
\hline T1DM & 1 \\
\hline \multicolumn{2}{|l|}{ Primiparous } \\
\hline Yes & 4 \\
\hline
\end{tabular}

Person who advised woman to collect antenatal colostrum*

\begin{tabular}{ll} 
Midwife & 5 \\
\hline Lactation consultant & 5 \\
\hline Diabetic educator & 3 \\
Obstetrician & 2 \\
Other† & 3 \\
Woman obtained antenatal colostrum & \\
$\quad$ Yes & 5 \\
\hline Baby became hypoglycaemic & \\
$\quad$ Yes & 4 \\
\hline Treatment of hypoglycaemia* & \\
$\quad$ EBM & 4 \\
\hline Formula & 4 \\
\hline Intravenous dextrose & 1 \\
\hline
\end{tabular}

*More than one answer given.

†Other included Breastfeeding Australia, friends and general practitioners.

EBM, expressed breast milk; GDM, gestational diabetes mellitus; T1DM, type I diabetes mellitus.

\section{Themes}

Six major themes emerged: wary of medicalisation, underlying altruism, internal pressure to succeed, self-management and ownership, frustration with waste and building fortitude for motherhood. Each theme and corresponding subthemes are outlined below. Illustrative quotes are provided in table 2 . Figure 1 depicts a thematic schema which maps conceptual links between themes.
WARY OF MEDICALISATION

\section{Adjusting to an 'abnormal pregnancy'}

Participants reflected that having diabetes was an 'added complication' that made their pregnancy unnatural. They felt 'shocked' and 'terrified' when they were diagnosed with diabetes and were especially concerned about the added risks to their baby. Some felt distressed to be labelled a 'high-risk pregnancy', especially when they had been 'fit and well' prior to diagnosis.

\section{Seeking continuity of care}

Participants emphasised the importance of having continuity of care throughout their pregnancy. They were disappointed when they were moved from a low-risk midwife clinic to the diabetes clinic where every week they would 'see someone new'. They strove to maintain control of their diabetes to avoid changes to their care-'If I didn't manage the diabetes I wasn't going to be able to give birth at the birth centre.'

\section{Determination to reduce formula}

Participants actively sought to avoid formula, iterating the benefits of breast feeding over formula use-'It is better than formula'. They felt that by collecting and storing colostrum, they were able to ensure that formula consumption could be reduced. Women whose babies experienced hypoglycaemia were 'overwhelmed' by how much formula was used, which they considered was unnatural and invasive as it was 'pouring down the tube'.

\section{Fear of invasive intervention}

Throughout pregnancy, participants experienced relentless fear that their babies and they might be subjected to invasive interventions. They feared missing out on a 'natural, empowered birth' and were particularly frightened by the idea that their baby might require nasogastric tubing or intravenous cannulation. They described feeling 'heartbroken' when their babies were taken away from them to the special care nursery (SCN).

\section{Underlying altruism}

Providing the best for baby

Participants were motivated to collect and store colostrum by their ingrained desire to provide 'the best' for their babies. They described that colostrum could provide 'extra nutrients' and 'immunity' and that it had immeasurable benefits in the first hours and days. Some participants stated that collecting colostrum had no benefits to themselves but emphasised that 'you don't go into motherhood to think about yourself'. Ultimately, they believed that by having collected colostrum available, they were ensuring their baby had the best possible start-'If it's the best start for a baby, let alone a gestational diabetic baby, I wanted to do anything that I could do to help him!'

\section{Preparing for complications}

Participants perceived that complications, especially neonatal hypoglycaemia, were inevitable, a 'sure thing' and that the threat of these complications made collecting 
Table 2 Themes and illustrative quotes

Theme Illustrative quotes

Wary of medicalisation

Adjusting to an 'abnormal pregnancy'

When you're pregnant, you're trying to figure it out anyway and then you've got the added complication of diabetes. (GDM, 20s)

I cried on the phone ... I was like, 'I don't know what I'm doing, I don't know what's going on!' (T1DM, 20s)

Seeking continuity of care The continuity of care is so important! (GDM, 20s)

Determination to reduce formula We managed to avoid a few top up feeds from the formula. (GDM, 20s)

Get as much as you can! Because it is better than formula! (T1DM, 20s)

Fear of invasive intervention It heartbreaking actually because they ended up having to put in drips and things to stabilise it [BGL]. (GDM, 40s)

Underlying altruism

Providing the best for baby It was an important thing to do for the baby so you just do it. (GDM, 40 s)

The milk beforehand was so good for the baby. (GDM, 30s)

Preparing for inevitable I knew very early that l'd be having a (caesarean section). So should've anything

complications

happen there was that (colostrum) there. (GDM, 30s)

Eager for milk donation There was a mother struggling and she didn't have any and I couldn't have been happier to give her some of mine. (GDM, 30s)

Even if your baby doesn't need it, there's possibilities that other babies would be able to use it as well. (GDM, 40s)

Internal pressure to succeed

Coping with confronting information It's just an overwhelming lot of information. (GDM, 20s)

Disheartened by failures I was just one of those women that didn't get a colostrum. I tried so hard! (GDM, 20s)

I tried a bit earlier on and I was unsuccessful to get anything. (GDM, 30s)

Constant fear of insufficient supply I just kept looking at all the packets of stuff that I hadn't filled and thought 'I've just got to get these filled'. (GDM, 30s)

I remember him (obstetrician) going 'Calm down he'll be fine' I'm like, 'I'm freaking out, I don't have any colostrum!' (GDM, 20s)

Overwhelming guilt If I didn't do it and she needed it, l'd only have myself to blame if it wasn't there. (GDM, 30s)

I just felt that my body could give more. I just didn't put the time in. (T1DM, 20s)

Concern for future breastfeeding I was beginning to actually get scared I would never make milk. (GDM, 20s)

success

Frustrated by waste

Encroaching on time It (expressing) was taking me probably a couple of hours to do a night. (GDM, 30s)

So yeah it (expressing) was just time consuming. (GDM, 30s)

Squandering a precious resource I showed up with $20 \mathrm{mls}$ of colostrum thinking I' m sweet and disappeared in three feeds! (GDM, 20s)

It seemed like a waste that it [colostrum] eventually went in the bin.(GDM, $30 \mathrm{~s}$ )

Ambiguous about necessity I didn't end up using the milk. My daughter didn't need it. (GDM, 30s)

It was just quite ambiguous.... Why am I really doing this? (T1DM, 20s)

Self-management and ownership

Adapting to awkwardness

I felt like it [expressing] was a bit weird. Then when I started to collect I was like 'Okay I get it'. (T1DM, 20s)

It didn't seem odd or unusual, just natural. (GDM, 40s)

Developing strategies for success I had specimen cups that I collected in and I had all the syringes and had caps and all the labels already printed. (GDM, 30s)

I used warm compresses and made sure that I was nice and warm as well. And I would heat up a flannel and pop that over my breast before I tried to express. (GDM, $40 \mathrm{~s}$ )

Actively seeking education

I YouTubed, the best way to actually collect. (GDM, 30 s)

I did some research on it and I saw the benefits. (T1DM, 20s)

Continued 


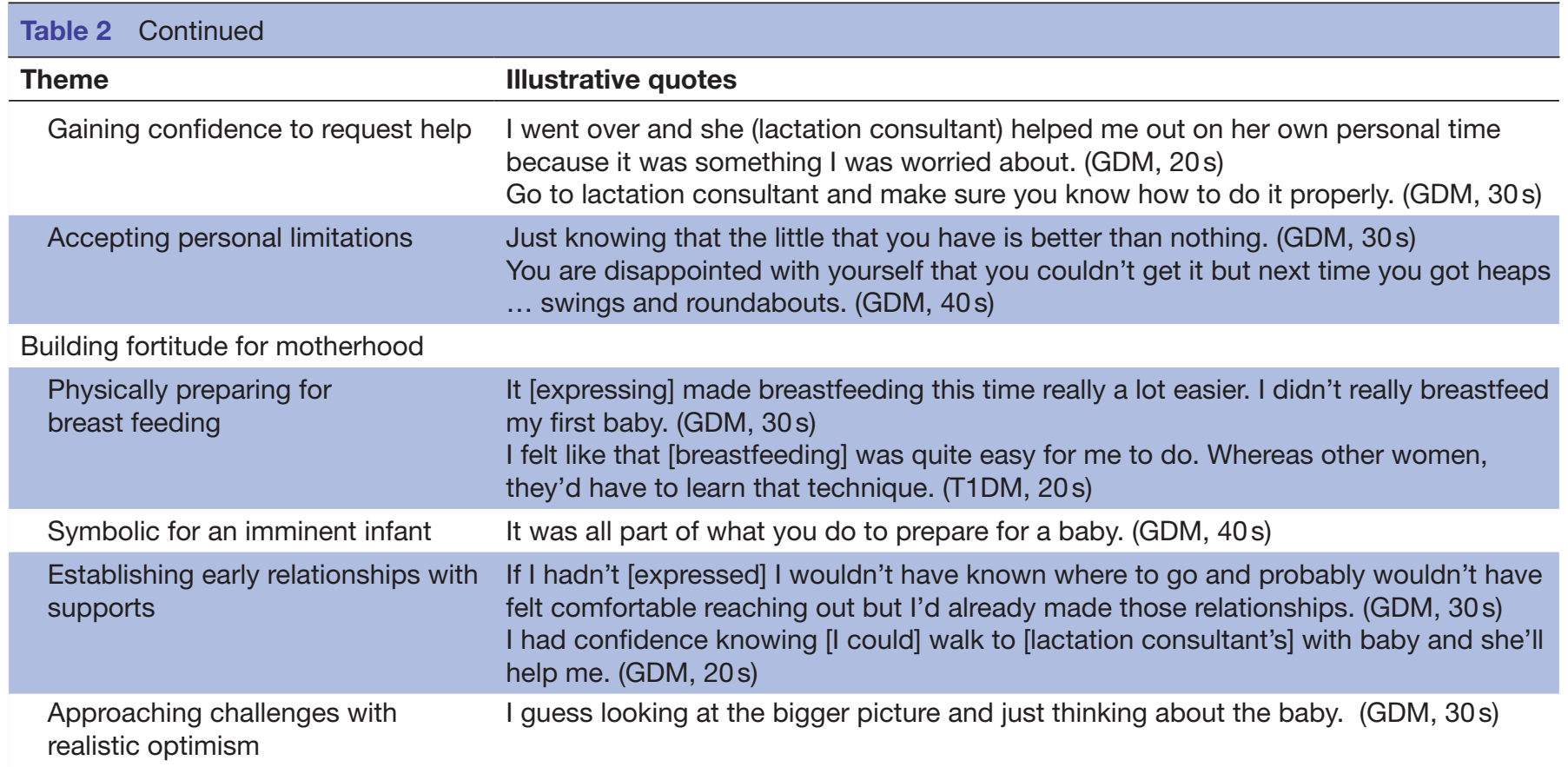

BGL, blood glucose level; GDM, gestational diabetes mellitus; T1DM, type I diabetes mellitus.

colostrum so important. They felt more secure knowing that the colostrum was available as a 'safety net or a backup' and described a sense of pride and confidence at seeing the syringe in the freezer 'ready to go' in case the baby experienced low blood sugars or need to be taken to SCN. Collecting was a tangible strategy to 'be prepared for the worst, if it should happen.'

\section{Eager for milk donation}

For many participants, altruism extended beyond their own babies, as they expressed strong desire for opportunities to donate their collected colostrum. Women who had a plentiful supply of collected colostrum were saddened that there was no milk bank they could donate it to, especially when they saw other mothers struggling. Similarly, women who had been unable to obtain colostrum were devastated that they were 'not allowed to bring in donated breast milk'. Some women even thought they would have been motivated to collect more colostrum if a milk bank or similar capability was available because of the possibilities that other babies could use it as well.

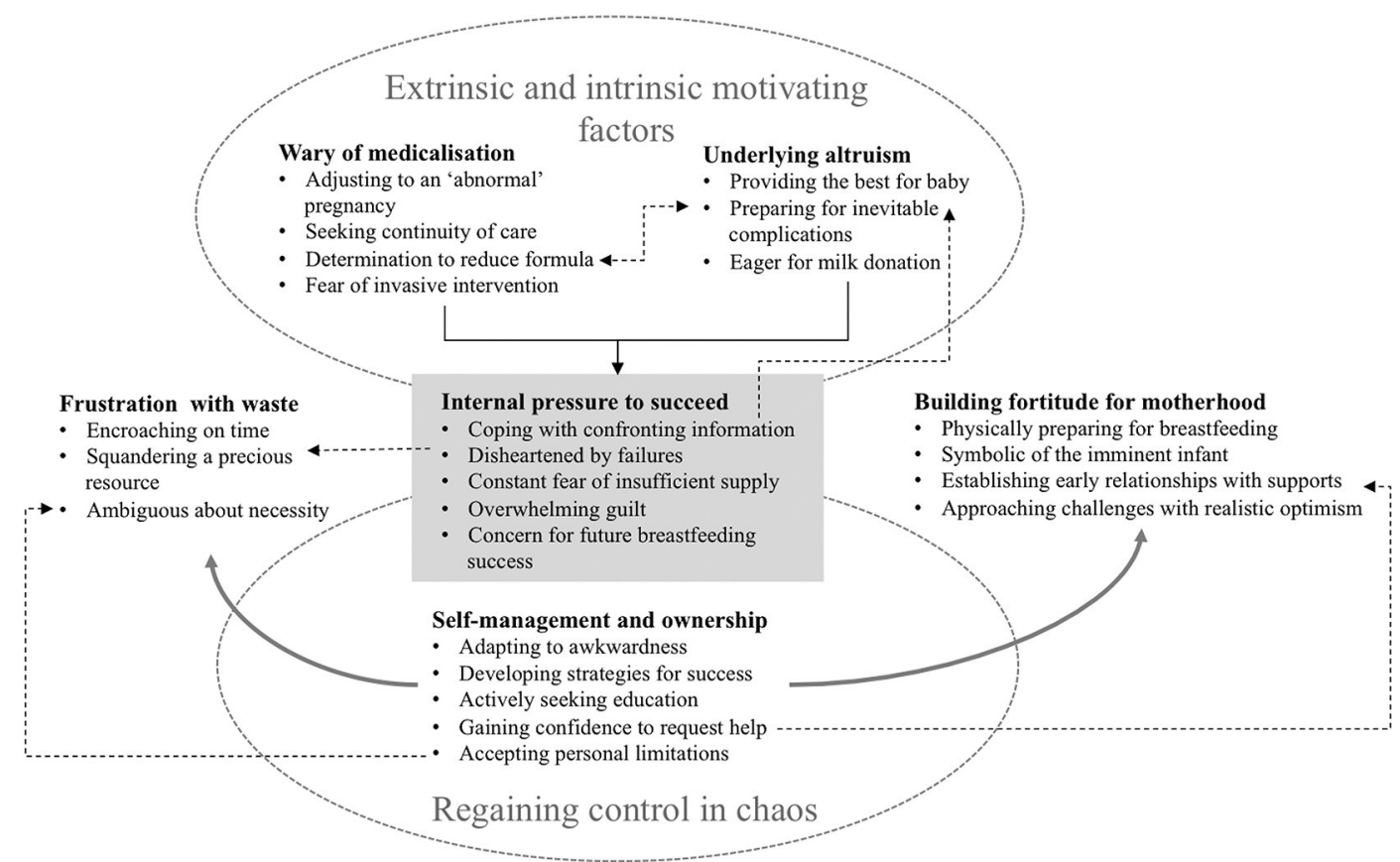

Figure 1 Thematic schema of diabetic women's experiences of collecting colostrum in pregnancy. 


\section{Internal pressure to succeed}

Coping with confronting information

The amount and content of information provided during pregnancy was overwhelming for many participants. Some 'never realised' that it was possible to express breast milk prior to birth and that they would have to do it. Women recounted being asked how they would feel if their babies required interventions to correct their blood sugars and found these questions confronting-'I was even told that diabetics have issues with milk supply. They said to me "How are you going to feel if he has to go on formula?"

\section{Disheartened by failures}

Participants described a sense of failure when they have difficulties collecting colostrum and were 'devastated' when they were unable to obtain colostrum. They experienced ongoing worry that they were using an incorrect technique and found that the process continued to be frustrating and stressful even after they had successfully managed to collect some colostrum. Some women questioned whether the process was so difficult because they were 'forcing it to come out when it's not fully ready'. Women who had ongoing difficulties collecting colostrum became particularly distressed by their perceived failure-'I went down there just balling!'

\section{Constant fear of insufficient supply}

Many participants experienced ongoing and often relentless anxiety that they would have insufficient colostrum collected for their baby. Some described looking at the empty syringes and feeling pressured and panicked to 'get these filled!'. Though many women were able to successfully collect multiple syringes of colostrum, they remained absorbed by the idea that they were not getting enough and that the amount they had was 'nothing compared' to what they perceived they needed. Some participants compared their supply of colostrum to other expressing women and were uneasy that they had not collected as much as others. For some women, the lack of colostrum supply led them to approach the birth of their baby with extreme apprehension- 'They were talking about induction and I said 'But I don't have any colostrum yet!"

\section{Overwhelming guilt}

Having diabetes in pregnancy evoked a sense of guilt for many participants, especially because of its implications for their babies and the 'many extra risks' it added. This guilt extended to collecting colostrum as women experienced disappointment and self-blame when they struggled with the process. Some women worried that their technique could make the collected colostrum harmful to their baby such as difficulties with sterilisation. Women felt remorseful that they had not started collecting earlier in their pregnancy and some lamented that they did not dedicate more time and effort to the process.

\section{Concern for future breastfeeding success}

For some participants, difficulties with collecting colostrum in the antenatal period led them to question whether they would have success with breast feeding postnatally. They became stressed that their lack of colostrum was a reflection of their future postnatal lactational performance-'I was beginning to actually get scared that I was never going to make milk!'

\section{Self-management and ownership}

Adapting to awkwardness

To enable the collection of colostrum, participants were required to adapt to a process that many found 'weird' and 'funny' and associated with underlying embarrassment. However, participants explained that once they began to express they gained more confidence, and experienced pride and excitement when they saw how much they were collecting. Ultimately they were able to accept that the process was 'not odd or unusual, just natural.'

\section{Developing strategies for success}

Many participants were empowered to develop personal strategies to enable them to collect and store more colostrum. They described using methods such warm compresses, showers and relaxation techniques to help initiate colostrum expression and experimented with collecting instruments including syringes, teaspoons, collection cups and mechanical breast pumps. Ultimately, many women perceived that the key to obtaining colostrum was organisation and relaxation-'When you got a routine down it became quite easy.'

\section{Actively seeking education}

Participants sought to become more educated about collecting colostrum antenatally by using social media groups, watching YouTube videos and seeking written material from their midwives and lactation consultants. Some women thought the amount of education provided could be improved, for example, by using case studies to emphasise why collecting colostrum might be important for the mother and baby.

\section{Gaining confidence to request help}

Participants enthusiastically communicated the role that supports such as midwives and lactation consultants played in providing advice and encouragement to help them with collecting. Some women also relied on family such as their mother or spouse to provide support and one woman contacted Breastfeeding Australia to gain further reassurance. Women who had difficulties collecting colostrum felt confident after gaining professional help from a lactation consultant-'I didn't get any colostrum but it felt good because someone else had tried to do it.'

\section{Accepting personal limitations}

Participants were able to overcome their personal limitation with collecting colostrum and begin to take pride in small successes. They acknowledged that the process could be 'luck of the draw' and they need to celebrate the 'little wins'. For many, positive thinking was the mainstay of adjusting to diabetes in pregnancy and collecting 
colostrum-'Some' days you've just got to chalk it up to a bad day and try again tomorrow.'

\section{Frustration with waste}

\section{Encroaching on time}

Collecting colostrum was pronounced a tedious and time-consuming process that participants reported could take hours to complete. Some participants also felt that appointments associated with collecting colostrum, such as visiting the lactation consultant, took up excessive time, especially given the many appointments they were required to attend because of their diabetes. One woman thought it would be particularly difficult for working women-'I was lucky because I didn't work, so I could come to the hospital a lot more.'

\section{Squandering a precious resource}

Participants found it was easy to waste colostrum and were frustrated when they missed drops when expressing or left colostrum on the teaspoon. They were also extremely disappointed when their supply of collected colostrum was wasted if they didn't need to use it, especially when it 'eventually went in the bin.' Women with both ample and insufficient supply of colostrum reiterated their disappointment that there are no opportunities for milk donation, to prevent the expressed colostrum from merely being 'dumped'.

\section{Ambiguous about necessity}

Some participants questioned whether having colostrum available was really worth the effort required to collect it. They found themselves asking-'Why am I really doing this?'

\section{Building fortitude for motherhood \\ Preparing for breast feeding}

Participants emphasised that collecting colostrum antenatally helped them prepare for breast feeding postnatally. Some participants noticed that their milk 'came on a lot quicker' than with previous pregnancies. Others felt the skills they had learnt to hand express were useful when their baby needed tops-up to supplement breast feeding. Expressing in the antenatal period also provided mental fortitude for breast feeding-'I know I can make milk, it's in my head.'

\section{Symbolic of the imminent infant}

Collecting colostrum was a tangible symbol of the coming baby for many participants, a reminder that they would soon be mothers and a practical way to 'prepare' for a baby-'Expressing was the first time that it was like, you're having a baby!'

\section{Establishing early relationships with breastfeeding supports}

By seeking advice as they negotiated the challenges of collecting antenatal colostrum, participants were able to build relationships with professionals and organisations that could provide support throughout breast feeding. They perceived that expressing helped them know who to ask for help and gave them the confidence to admit when they were having difficulties postnatally.

Approaching challenges with realistic optimism

Participants felt that the psychological strategies they developed to cope with the frustrations of diabetes in pregnancy, and especially collecting colostrum, ultimately aided them to approach the inevitable challenges of motherhood with similar positivity-'I felt like it [collecting colostrum] prepared me, hardened everything up.'

\section{DISCUSSION}

In this analysis of the experiences of women with diabetes in pregnancy, women were motivated to collect and store colostrum by wariness of medicalisation and an underlying desire to provide the best start for their babies and avoid unnecessary interventions such as formula. These motivating factors, however, also contributed to an ongoing and often overwhelming pressure to obtain colostrum, associated with considerable guilt and distress when they had difficulties with the process. This pressure also drove women to develop personal strategies to regain control in the context of their pregnancy complicated by diabetes. Women were able to adjust to frustrations associated with expressing and ultimately develop biopsychosocial fortitude for motherhood.

One major finding that emerged in this study was participants' eagerness for the availability of milk banks, as a strategy to reduce wastage of unused colostrum and as an outlet of altruism to support other mothers with an insufficient supply. A recent qualitative study of mother's opinions of human milk banking found that women perceived donor milk as healthier than formula and that it allowed smoother transition to exclusive breast feeding. ${ }^{14}$ Donor breast milk has been shown to offer health benefits compared with formula including reduction in complications such as necrotising enterocolitis and an improvement in feeding tolerance. ${ }^{15}{ }^{16}$ Given that women dedicate considerable time and effort to collecting colostrum, which often goes unused, protocols should be in place to minimise waste, such as through donation, if this practice continues to be promoted.

Women in this study who were able to collect colostrum in the antenatal period perceived that they were more physically and psychosocially prepared for breast feeding postnatally. Similarly, previous qualitative analysis of women without diabetes in pregnancy revealed that antenatal expressing can bolster women's confidence in breast feeding by facilitating a sense of mastery and breast familiarity. ${ }^{11}$ Breast feeding is particularly beneficial for mothers with diabetes and their newborns because it can reduce the development of type II diabetes in mothers with GDM and can reduce the development of diabetes in the child in the long term and hypoglycaemia in the short term. ${ }^{17-19}$ However, diabetes in pregnancy has been associated with delayed lactogenesis, which paradoxically makes breast feeding more difficult for these mothers. ${ }^{20}$ 
A recent prospective study in a non-diabetic population found that expressing antenatally can significantly improve postnatal lactational performance by early establishment of plentiful milk secretion. ${ }^{10}$ Further investigation of the effects of collecting colostrum antenatally on time to full lactation in the diabetic population appears warranted.

We performed in-depth, face-to-face interviewing which allowed for a detailed understanding of women's experiences of collecting and storing colostrum. Participants were interviewed until no new concepts emerged (ie, theoretical saturation was achieved). Researcher triangulation was used to ensure the themes reflected the range and depth of data collected. However, this study had some limitations. Non-English speaking participants were excluded from the study and only women of Caucasian ethnicity were represented in the study sample. Participants were only recruited from one site so the transferability to other settings is uncertain.

\section{CONCLUSION}

Women who have diabetes in pregnancy experience stress and guilt due to the added risks to their babies, especially neonatal hypoglycaemia, and experience internal pressure to provide the best for their babies by collecting colostrum, even though this process can cause further distress and frustration. Though the DAME trial found that expressing antenatally is safe for low-risk diabetic women, no specific benefits to the newborn have been demonstrated. Continuing to place undue emphasis on the benefits of collecting and storing colostrum to the neonate may have the potential to cause unnecessary stress, guilt and ultimately harm to women already coping with the stress of diabetes in pregnancy. Care must be taken to present accurate and realistic recommendations to women with diabetes in pregnancy about collecting antenatal colostrum to facilitate informed decision-making.

Acknowledgements The authors acknowledge Lisa Smith for her assistance with participant recruitment. We also particularly acknowledge the women who gave up their time to participate in this study.

Contributors JRRC designed the study, conducted the interviews, coded and analysed the data, carried out the thematic analysis and drafted the manuscript. ELM coded and analysed the data, participated in the thematic analysis and critical review of the first and subsequent manuscript drafts. JB assisted with study design and interview tool development, provided intellectual input into first and subsequent manuscript drafts and assisted with thematic analysis. KB conceived the study, assisted with study design and interview tool development and provided intellectual input into subsequent manuscript drafts. $\mathrm{CH}$ assisted with study design and interview tool development, provided intellectual input into first and subsequent manuscript drafts, assisted with thematic analysis and is the guarantor. All authors had full access to all of the data in the study and can take responsibility for the integrity of the data and the accuracy of the data analysis.

Funding This work was supported by the College of Medicine and Dentistry, James Cook University (grant number JCU-QLD-578961).

Competing interests None declared.

Patient consent for publication Not required.
Ethics approval Ethics approval was obtained for this study from The Townsville Hospital and Health Service (HREC/16/QTHS/66).

Provenance and peer review Not commissioned; externally peer reviewed.

Data sharing statement № additional data are available.

Open access This is an open access article distributed in accordance with the Creative Commons Attribution Non Commercial (CC BY-NC 4.0) license, which permits others to distribute, remix, adapt, build upon this work non-commercially, and license their derivative works on different terms, provided the original work is properly cited, appropriate credit is given, any changes made indicated, and the use is non-commercial. See: http://creativecommons.org/licenses/by-nc/4.0/.

\section{REFERENCES}

1 Moses RG, Wong VC, Lambert K, et al. The prevalence of hyperglycaemia in pregnancy in Australia. Aust N Z J Obstet Gynaecol 2016;56:341-5.

2 Metzger BE, Persson B, Lowe LP, et al. Hyperglycemia and adverse pregnancy outcome study: neonatal glycemia. Pediatrics 2010;126:e1545-52.

3 Mitanchez D, Yzydorczyk C, Simeoni U. What neonatal complications should the pediatrician be aware of in case of maternal gestational diabetes? World J Diabetes 2015;6:734-43.

4 Kaiser JR, Bai S, Gibson N, et al. Association between transient newborn hypoglycemia and fourth-grade achievement test proficiency: a population-based study. JAMA Pediatr 2015;169:913-21.

5 Maayan-Metzger A, Lubin D, Kuint J. Hypoglycemia rates in the first days of life among term infants born to diabetic mothers. Neonatology 2009;96:80-5.

6 Cox SG. Expressing and storing colostrum antenatally for use in the newborn period. Breastfeed Rev 2006;14:11-16.

7 Oza-Frank R, Moreland JJ, McNamara K, et al. Early lactation and infant feeding practices differ by maternal gestational diabetes history. J Hum Lact 2016;32:658-65.

8 Lamba S, Chopra S, Negi M. Effect of antenatal breast milk expression at term pregnancy to improve post natal lactational performance. J Obstet Gynaecol India 2016;66:30-4.

9 Della FA, Anita M, Susan J, et al. Is not testing a major change in clinical practice an ethical dilemma? Midwifery 2011;27:e301-2.

10 Forster DA, Moorhead AM, Jacobs SE, et al. Advising women with diabetes in pregnancy to express breastmilk in late pregnancy (Diabetes and Antenatal Milk Expressing [DAME]): a multicentre, unblinded, randomised controlled trial. Lancet 2017;389(10085):2204-13.

11 Brisbane JM, Giglia RC. Experiences of expressing and storing colostrum antenatally: A qualitative study of mothers in regional Western Australia. J Child Health Care 2015;19:206-15.

12 Tong A, Sainsbury P, Craig J. Consolidated Criteria for Reporting Qualitative Research (COREQ): a 32-item checklist for interviews and focus groups. Int J Qual Health Care 2007;19:349-57.

13 Straus A, Corbin J. Basics of qualitative research. Techniques and procedures for developing grounded theory. Thousand Oaks, California: Sage Publications, 1998.

14 Mackenzie C, Javanparast S, Newman L. Mothers' knowledge of and attitudes toward human milk banking in South Australia: a qualitative study. J Hum Lact 2013;29:222-9.

15 Quigley M, McGuire W. Formula versus donor breast milk for feeding preterm or low birth weight infants. Cochrane Database Syst Rev 2014;4:Cd002971.

16 Arslanoglu S, Moro GE, Bellù R, et al. Presence of human milk bank is associated with elevated rate of exclusive breastfeeding in VLBW infants. J Perinat Med 2013;41:129-31.

17 Chertok IR, Raz I, Shoham I, et al. Effects of early breastfeeding on neonatal glucose levels of term infants born to women with gestational diabetes. J Hum Nutr Diet 2009;22:166-9.

18 Much D, Beyerlein A, Roßbauer M, et al. Beneficial effects of breastfeeding in women with gestational diabetes mellitus. Mol Metab 2014;3:284-92.

19 Pereira PF, Alfenas RC, Araújo RM. Does breastfeeding influence the risk of developing diabetes mellitus in children? A review of current evidence. J Pediatr 2014;90:7-15.

20 De Bortoli J, Amir LH. Is onset of lactation delayed in women with diabetes in pregnancy? A systematic review. Diabet Med 2016;33:17-24. 